\title{
Beclomethasone/Formoterol in Extra-Fine Formulation Improves Small Airway Dysfunction in COPD Patients
}

\author{
Roberta Pisi · Marina Aiello (D) - Alessio Piraino · Davide Paleari • \\ Annalisa Frizzelli · Giuseppina Bertorelli · Alfredo Chetta
}

Received: August 3, 2020 / Accepted: January 9, 2021 / Published online: February 4, 2021

(C) The Author(s) 2021

\begin{abstract}
Introduction: In patients with chronic obstructive pulmonary disease (COPD), small airway dysfunction (SAD) is a key element and a functional consequence of the pathology. The exact role of SAD as a specific 'pharmacological target' represents an important research topic. Our objective was to ascertain whether an extrafine formulation of beclomethasone dipropionate/formoterol fumarate (BDP/FF) NEXThaler ${ }^{\circledR} 100 / 6 \mu$ g b.i.d. could improve SAD and, consequently, the quality of life of COPD patients.

Methods: We enrolled COPD patients with severe airflow obstruction and at least one moderate exacerbation in the previous year, having started treatment with BDP/FF NEXThaler $^{\circledR}$ for no more than 1 week. Patients underwent three visits: at the start of the treatment (V1), 6 weeks (V2), and 12 weeks later (V3). At each visit, we evaluated the fall in resistance from 5 to $20 \mathrm{~Hz}$ (R5-R20) and residual
\end{abstract}

R. Pisi · M. Aiello ( $₫)$ · A. Frizzelli · G. Bertorelli ·

A. Chetta

Respiratory Disease and Lung Function Unit,

Department of Medicine and Surgery, University of Parma, Parma, Italy

e-mail: marina.aiello@unipr.it

A. Piraino · D. Paleari

Medical Affairs, Chiesi Italy, Chiesi Farmaceutici

S.P.A., Parma, Italy volume/total lung capacity (RV/TLC) ratio by impulse oscillometry, spirometry, and plethysmography. The COPD Assessment Test (CAT) and the modified Medical Research Council (mMRC) questionnaire were also administered to assess the disease's impact on quality of life. Results: We enrolled 43 COPD patients (mean age 69 years, $\mathrm{FEV}_{1} 43 \%$ ). R5-R20 significantly changed from baseline $[0.23 \pm 0.09 \mathrm{kPa} /(\mathrm{l} / \mathrm{s})]$ to V2 $[0.16 \pm 0.09 \mathrm{kPa} /(1 / \mathrm{s})] \quad$ and $\mathrm{V} 3$ $[0.16 \pm 0.08 \mathrm{kPa} /(\mathrm{l} / \mathrm{s})](p<0.05)$. Clinical status was also significantly improved compared to baseline; in fact, CAT score changed from an average baseline value of 13-6 and 4 (V2 and $\mathrm{V} 3$, respectively) $(p<0.05)$. A correlation was found between CAT percentage change values and the corresponding ones of R5-R20 $(r=-$ $0.329, p=0.045)$ and RV/TLC $(r=0.354$, $p=0.029)$.

Conclusions: In COPD patients, treatment with $\mathrm{BDP} / \mathrm{FF}$ extra-fine formulation improved functional parameters related to small airway disease as well as the disease impact on health status.

Trial Registration: ClinicalTrials.gov identifier, NCT04421742.

Keywords: Beclomethasone/formoterol; COPD; Small airway dysfunction 


\section{Key Summary Points}

Why carry out this study?

Small-airway dysfunction (SAD) plays a key role in the pathogenesis of COPD.

The study investigated if an extra-fine formulation of beclomethasone dipropionate/formoterol fumarate, designed to target the entire bronchial tree, including the peripheral zone, can improve the impact of the disease on the quality of life of COPD patients, by acting on SAD.

\section{What was learned from the study?}

Treatment with BDP/FF extra-fine formulation improved functional parameters related to small airway disease and the disease impact on health status.

This study shows a link between the effect of the extra-fine BDP/FF DPI combination on the small airways and improvement in the symptoms of COPD patients.

BDP/FF delivered in an extra-fine formulation may be considered a valid combination therapy for a wide population of symptomatic COPD patients.

\section{DIGITAL FEATURES}

This article is published with digital features, including a summary slide, to facilitate understanding of the article. To view digital features for this article go to https://doi.org/10.6084/ m9.figshare.13415903.

\section{INTRODUCTION}

In recent years, there has been growing interest in deepening our understanding of how small airways (with a diameter of less than $2 \mathrm{~mm}$ ) contribute to the clinical expression of chronic obstructive pulmonary disease (COPD). The GOLD document (Global Initiative for Chronic Obstructive Lung Disease) defines COPD's pathophysiology by linking flow limitation to a mixture of small airway disease, obstructive bronchiolitis, and parenchymal destruction and emphysema [1]. In COPD, the extension of the inflammatory process, fibrosis, and endoluminal exudate determine small airway dysfunction (SAD), which is considered a key element and functional consequence of the pathology $[2,3]$.

In patients with stable COPD, SAD severity was progressively increased with the degree of airflow obstruction and GOLD risk class [4]. Moreover, a correlation between SAD and COPD impact, measured with the COPD Assessment Test (CAT), was found. The severity of SAD was a predictor of the impact of the disease (CAT $\geq 10$ ). A close correlation between SAD and both quality of life (measured with the St. George's Respiratory Questionnaire-SGRQ questionnaire) and perceived dyspnea (measured with the modified Medical Research Council-mMRC scale) was also found in COPD patients [5].

The exact impact of SAD as a specific 'pharmacological target' is not yet fully understood. According to the guidelines, the administration of a drug combination such as beclomethasone dipropionate/formoterol fumarate (BDP/FF) in extra-fine formulation produces a clinically relevant improvement in the quality of life $(>4$ units in the SGRQ questionnaire) and dyspnea $[6,7]$. However, it is unclear if a correlation between symptomatic improvement and SAD exists, and whether pharmacological therapy directed toward small airways can improve symptoms.

The rationale for this study is based on the hypothesis that an extra-fine formulation of $\mathrm{BDP} / \mathrm{FF}$, designed to target the entire bronchial tree, including the peripheral zone, can improve the disease's impact on the quality of life of COPD patients by acting on SAD. The functional indices of SAD used in this study are derived from the impulse oscillometry system (IOS), standard spirometry, and body plethysmography measurements. The primary endpoint of the study was, therefore, an 
improvement in the quality of daily life (measured by CAT) and dyspnea (mMRC) after 12 weeks of treatment with an extra-fine formulation of $\mathrm{BDP} / \mathrm{FF}$ in a cohort of COPD patients with severe airway obstruction and one moderate exacerbation in the previous year. The secondary endpoint was an improvement in lung function parameters of SAD at week 12 in these patients.

\section{METHODS}

\section{Participants and Study Design}

This pilot, prospective, open-label study was conducted at the outpatient clinic of the Respiratory Disease Unit of the University Hospital in Parma (Italy) over 20 months between April 2017 and March 2019. We enrolled patients: (a) of both sexes, aged $\geq 40$ years; (b) with a documented diagnosis of COPD defined according to the guidelines of the American Thoracic Society (ATS)/European Respiratory Society (ERS) [8]; (c) current or former smokers with a smoking history of at least ten pack/years (d) subjects with a post-bronchodilator forced expiratory volume at the first second $\left(\mathrm{FEV}_{1}\right)$ / forced vital capacity (FVC) ratio of $<0.7$ and an $\mathrm{FEV}_{1}<50 \%$ predicted; (e) subjects with a high impact of the COPD on their health status (CAT $\geq 10$ ); (f) clinical history of at least one moderate exacerbation/year; (g) COPD patients being treated with BDP/FF NEXThaler ${ }^{\circledR} 100 / 6 \mu \mathrm{g}$ in extra-fine formulation no more than 1 week before enrollment.

We excluded patients with: (a) an exacerbation in the 4 weeks prior to enrollment; (b) other coexisting lung diseases (bronchial asthma, restrictive diseases, or bronchiectasis); (c) severe co-morbidities associated with COPD, such as uncontrolled cardiovascular disease, pneumonia or cancer; (d) pregnant patients; (e) subjects unable to meet the criteria of acceptability and repeatability of pulmonary function tests, according to the ATS/ERS document [9].

The BDP/FF extra-fine formulation (NEXThaler $^{\circledR}$, Chiesi Farmaceutici, Parma, Italy) $100 / 6 \mu \mathrm{g}$ was administered at the dosage of two inhalations b.i.d. for the study's whole duration

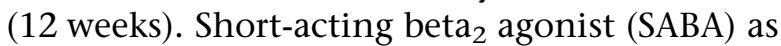
needed and concomitant medications for diseases other than COPD were allowed.

The study included three visits: V1 (screening), performed within the first week of treatment initiation with BDP/FF; V2 (after 6 weeks) and V3 (after 12 weeks). Visit 1 included: assessment of inclusion/exclusion criteria; signature of informed consent; the record of demographic and anthropometric variables (age, sex, and body mass index [BMI]), smoking habit (current/former), and number of packs per year, number of exacerbations in the year preceding the study; medical history investigation with the record of the disease duration, current therapies; co-morbidity evaluation through the Combined Comorbidity Index [10], COPD Assessment Test (CAT, Italian version) [11] to assess daily symptoms, Modified Medical Research Council dyspnea scale (mMRC, Italian version) for grading the effect of breathlessness on daily activities [12]; physical examination and record of vital parameters (respiration rate, heart rate, blood pressure, temperature, oxygen saturation); lung function testing (IOS, spirometry, and plethysmography),

visit 2 (after 6 weeks) and visit 3 (after 12 weeks) included: Medical history and physical examination; lung function testing (IOS, spirometry, and plethysmography); CAT; mMRC; Medication Adherence Report Scale (MARS-5) [13], a measure of adherence to BDP/ FF extra-fine formulation NEXThaler ${ }^{\circledR} 100 / 6 \mu \mathrm{g}$ (score 5-25; a score of 25 indicates perfect adherence). Adherence to inhalation treatment was also expressed as the percentage of the actual inhaled doses vs. the scheduled doses of BDP/FF extra-fine formulation NEXThaler ${ }^{\circledR}$ $100 / 6 \mu \mathrm{g}$.

The study protocol was approved by the Ethics Committee for northern Emilia, Italy (protocol number: 9769; approved on April 5, 2017), and conducted in accordance with good clinical practices and the Declaration of Helsinki. All enrolled patients gave their informed consent. 


\section{Spirometry and Plethysmography}

Spirometry was performed using a pneumotachograph connected to a computer for data analysis (Vyntus Spiro and Master Screen Body, Carefusion Germany $234 \mathrm{GmbH}$, Höchberg, Germany, EU). Forced vital capacity (FVC), forced expiratory volume at the first second $\left(\mathrm{FEV}_{1}\right)$, Tiffeneau Index $\left(\mathrm{FEV}_{1} / \mathrm{FVC}\right)$, and forced expiratory flow in the middle-half of the patient's exhaled volume (FEF25-75) were recorded. FVC, $\mathrm{FEV}_{1}$, and FEF25-75 were reported both in absolute value (liters, 1) and as a percentage of predicted $\mathrm{FEV}_{1} / \mathrm{FVC}$. Functional residual capacity (FRC) was measured by body plethysmography (Vyntus Spiro and Master Screen Body, Carefusion Germany $234 \mathrm{GmbH}$, Höchberg, Germany, EU). Total lung capacity (TLC) was the sum of FRC and linked inspiratory capacity (IC). The residual volume (VR) was calculated by subtracting the vital capacity (VC) from the TLC; RV to TLC (RV/TLC) and IC to TLC (IC/TLC) ratios were recorded.

At least three acceptable measurements were performed for each spirometry and lung volume variable to ensure reproducibility [14]. The spirometer and body plethysmograph were calibrated daily.

Lung diffusion capacity for carbon monoxide (TLco, \% pred) was measured by the single breath method using a mixture of carbon monoxide and methane; measurements were performed at least in duplicate.

Patients were advised to avoid any inhaled bronchodilators $12 \mathrm{~h}$ before the baseline spirometry. The reversibility test was performed with a second spirometry $15 \mathrm{~min}$ after administering inhaled salbutamol $(400 \mu \mathrm{g})$.

\section{Impulse Oscillometry System}

The IOS was performed with the Jaeger MasterScreen-IOS device (Carefusion Germany 234 GmbH, Höchberg, Germany, EU) following the standard recommendations [15]. Patients were asked to wear a nose clip during tidal breathing and hold their cheeks firmly with their hands to reduce upper airway shunting. At least three trials were performed, each lasting $30 \mathrm{~s}$, and the mean value was chosen. Respiratory resistance at $5 \mathrm{~Hz}$ and $20 \mathrm{~Hz}$ (R5 and $\mathrm{R} 20, \mathrm{kPa} \times \mathrm{s} \times \mathrm{l}^{-1}$ ) was used as total and proximal airway resistance index, respectively. The resistance drop from 5 to $20 \mathrm{~Hz}\left(\mathrm{R} 5-\mathrm{R} 20, \mathrm{kPa} \times \mathrm{s} \times \mathrm{l}^{-1}\right.$ ) was considered an indirect index of the peripheral airways' resistance. The $5-\mathrm{Hz}$ reactance $(\mathrm{X} 5, \mathrm{kPa} \times \mathrm{s} \times$ $\left.\mathrm{l}^{-1}\right)$ and reactance area $\left(\mathrm{AX}, \mathrm{kPa} \times \mathrm{l}^{-1}\right)$ were also measured because these are considered a hallmark of peripheral airways dysfunction [16]. The oscillometer was calibrated daily.

\section{Statistical Analysis}

Data were expressed as mean \pm standard deviation for continuous variables with a normal distribution and median [25th-75th percentile] for those with a non-normal distribution. The Shapiro-Wilk test was used to evaluate the normal distribution of each variable.

Comparisons were assessed by ANOVA and Bonferroni post hoc test for continuous variables with a normal distribution and by Kruskal-Wallis test and Dunn's multiple comparison test. Relationships between variables were assessed by Pearson correlation coefficient $(r)$ and linear regression analysis $\left(r^{2}\right)$.

A $p$ value $<0.05$ was considered significant.

\section{RESULTS}

We enrolled 43 patients with stable COPD (74\% males, mean age 69 years). At $\mathrm{V} 1$, the mean values of $\mathrm{FEV}_{1}, \mathrm{FEV}_{1} / \mathrm{FVC}, \mathrm{RV} / \mathrm{TLC}$ and R5-R20 were $43 \% \pm 8, \quad 49 \% \pm 9, \quad 59 \% \pm 7$ and $0.22 \pm 0.08 \mathrm{kPa} \times \mathrm{s} \times \mathrm{l}^{-1}$, respectively. In all patients, the R5-R20 value was greater than $0.07 \mathrm{kPa} \times \mathrm{s} \times \mathrm{l}^{-1}$, which is considered a cut-off value to define the presence of small airway dysfunction $[4,17]$. CAT and mMRC median values (25th-75th percentile) were 13 (11-17) and 1 (1-2), respectively. Clinical and functional data of the enrolled COPD patients are reported in Table 1.

Thirty-eight patients completed the study, and their adherence to the BDP/FF extra-fine formulation NEXThaler ${ }^{\circledR} 100 / 6 \mu \mathrm{g}$ was excellent both at V2 and V3. Median (25th-75th percentile) values of MARS-5 score and the 
Table 1 Demographic, clinical, and lung function characteristics of 43 COPD patients (12 females)

\begin{tabular}{|c|c|}
\hline Age (years) & $69 \pm 8$ \\
\hline BMI $\left(\mathrm{kg} / \mathrm{m}^{2}\right)$ & $26 \pm 4$ \\
\hline Pack/Years $(n)$. & $46 \pm 24$ \\
\hline Combined Comorbidity Index & $4[3-6]$ \\
\hline TLC (\% pred) & $114 \pm 20$ \\
\hline $\mathrm{FEV}_{1}(\%)$ & $43 \pm 8$ \\
\hline $\mathrm{FEV}_{1} / \mathrm{FVC}(\%)$ & $49 \pm 9$ \\
\hline $\mathrm{R} 5-\mathrm{R} 20\left(\mathrm{kPa} \times \mathrm{s} \times \mathrm{L}^{-1}\right)$ & $0.22 \pm 0.08$ \\
\hline IC/TLC (\%) & $28 \pm 8$ \\
\hline $\mathrm{TL}_{\mathrm{CO}}(\%$ pred $)$ & $62 \pm 14$ \\
\hline
\end{tabular}

Values are expressed as mean $\pm \mathrm{SD}$ or median $[25$ th -75 th percentile]

percentage of the actual inhaled doses vs. the scheduled doses of BDP/FF extra-fine formulation NEXThaler ${ }^{\circledR} 100 / 6 \mu$ g were 25 (22-25) and 98\% (74-100) at V2 and 24 (23-25) and 95\% (83-99) at V3, respectively. Patients had a median number of exacerbations of 1.00 (1-2) in the year before enrollment (range, 1-4). None of the patients enrolled in the study were under pharmacological treatment before enrollment. In most cases, patients started
NEXThaler treatment at V1 (screening/enrollment visit). Some patients had to learn how to use the device correctly and started treatment a few days later (but still within a week of V1). In all cases, we performed the first follow-up visit (V2) 6 weeks after starting treatment with NEXThaler. Five subjects did not complete the study for the following reasons: (1) the therapy was changed during the first 6 weeks of treatment; (2) hospitalization due to cardiac decompensation; (3) treatment-related side effects (oropharyngeal candidiasis, chills, sore throat, headache, asthenia, dizziness, anxiety, sleep disorders, gastrointestinal disorders), (4) and (5) hospitalization due to bronchial exacerbation.

Clinical (CAT and mMRC) and functional $\left(\mathrm{FEV}_{1}, \mathrm{RV} / \mathrm{TLC}, \mathrm{R} 5-\mathrm{R} 20, \mathrm{X} 5\right.$, and AX5) values were significantly improved at V2 and V3, as compared to the corresponding ones at V1 (Table 2 and Fig. 1). When the CAT score of V3 was compared to that of $\mathrm{V} 1$, an average decrease of 8 points was found. The reduction was greater than 2 points in all patients, which is considered the minimum clinically important difference [18]. Moreover, at V3, 32 out of 38 patients (84\%) had a CAT score of less than 10 , which denotes a low impact of COPD on a patient's life [11]. The percentage change values of V3 vs. V1 of CAT, R5-R20, and RV/TLC were $60 \% \pm 28, \quad 27 \% \pm 34, \quad 9 \% \pm 9, \quad$ respectively.

Table 2 Clinical and lung functional data of COPD patients at V1, V2, and V3

\begin{tabular}{llll}
\hline & V1 & V2 & V3 \\
\hline No & 43 & 38 & 38 \\
CAT $(0-40)$ & $13[11-17]$ & $6[4-9]^{*}$ & $4[3-7]^{*}$ \\
MRC $(0-4)$ & $1[1,2]$ & $1[0-1]^{*}$ & $1[0-1]^{*}$ \\
$\operatorname{FEV}_{1}(\%$ pred $)$ & $43 \pm 8$ & $49 \pm 13^{*}$ & $50 \pm 12^{*}$ \\
$\mathrm{FEV}_{1}(\mathrm{l})$ & $1.21 \pm 0.33$ & $1.43 \pm 0.43^{*}$ & $1.44 \pm 0.43^{*}$ \\
$\mathrm{RV} / \mathrm{TLC}(\%)$ & $59 \pm 7$ & $53 \pm 7^{*}$ & $53 \pm 7^{*}$ \\
$\mathrm{R} 5-20[\mathrm{kPa} /(1 / \mathrm{s})]$ & $0.23 \pm 0.09$ & $0.16 \pm 0.09^{*}$ & $0.16 \pm 0.08^{*}$ \\
$\mathrm{X} 5[\mathrm{kPa} /(1 / \mathrm{s})]$ & $-0.34 \pm 0.13$ & $-0.25 \pm 0.13^{*}$ & $-0.27 \pm 0.15^{*}$ \\
$\mathrm{AX5}[\mathrm{kPa} / \mathrm{l}]$ & $3.11 \pm 1.6$ & $1.98 \pm 1.5^{*}$ & $2.08 \pm 1.4$ \\
\hline
\end{tabular}

Values are expressed as mean $\pm \mathrm{SD}$ or median [25th-75th percentile]. ${ }^{*} p<0.05$ vs. V1 
A

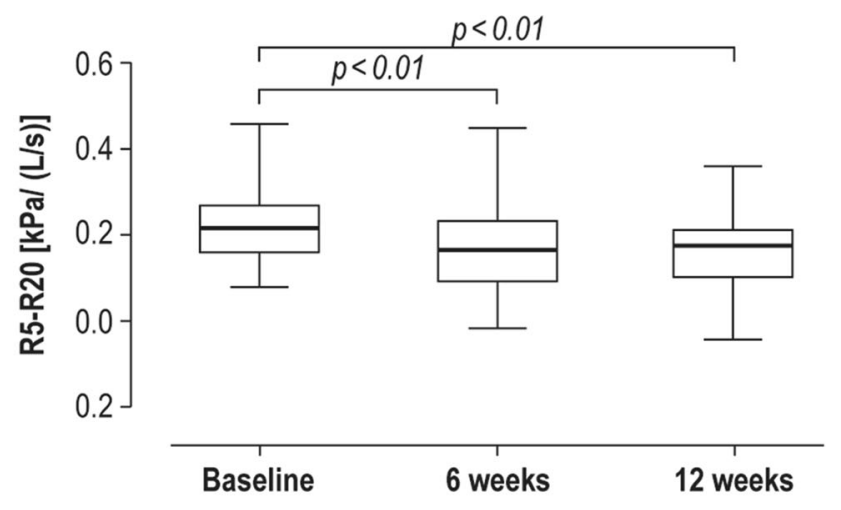

B $\quad p<0.01$

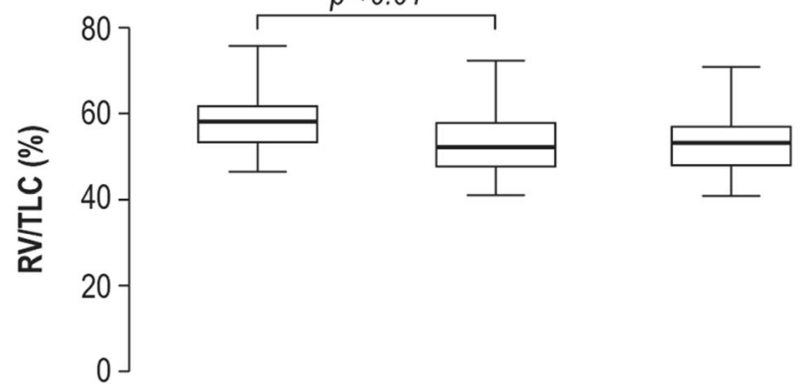

Baseline $\quad 6$ weeks 12 weeks

C

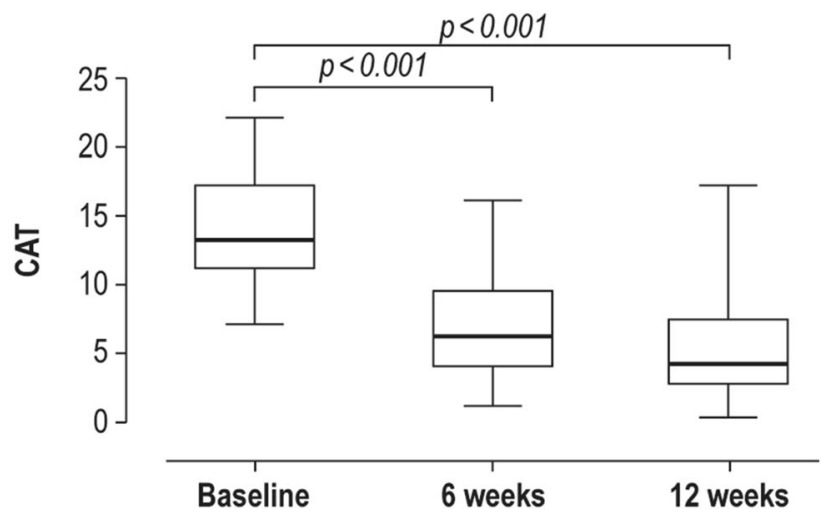

Fig. 1 Mean, standard deviation, and range of R5-R20 (a), RV/TLC (b), and CAT (c) at visit 1, 2, and 3 in the study population

CAT percentage change values were significantly correlated to the corresponding ones of R5-R20 $\left(r=-0.329, p=0.045 ; r^{2}=0.108\right)$ and $\mathrm{RV} / \mathrm{TLC} \quad\left(r=0.354, \quad \mathrm{p}=0.029 ; \quad r^{2}=0.125\right)$ (Fig. 2). R5-R20 percentage change values were also correlated to the corresponding ones of $\mathrm{RV} /$ TLC $\left(r=0.388, p=0.016 ; r^{2}=0.150\right)$ (Fig. 3).

\section{DISCUSSION}

The present prospective, open-label study supports the hypothesis that treatment with $\mathrm{BDP} /$ FF $100 / 6 \mu \mathrm{g}$ at a total daily dose of $400 / 24 \mu \mathrm{g}$ in an extra-fine formulation is able to significantly reduce distal airway resistance and pulmonary 

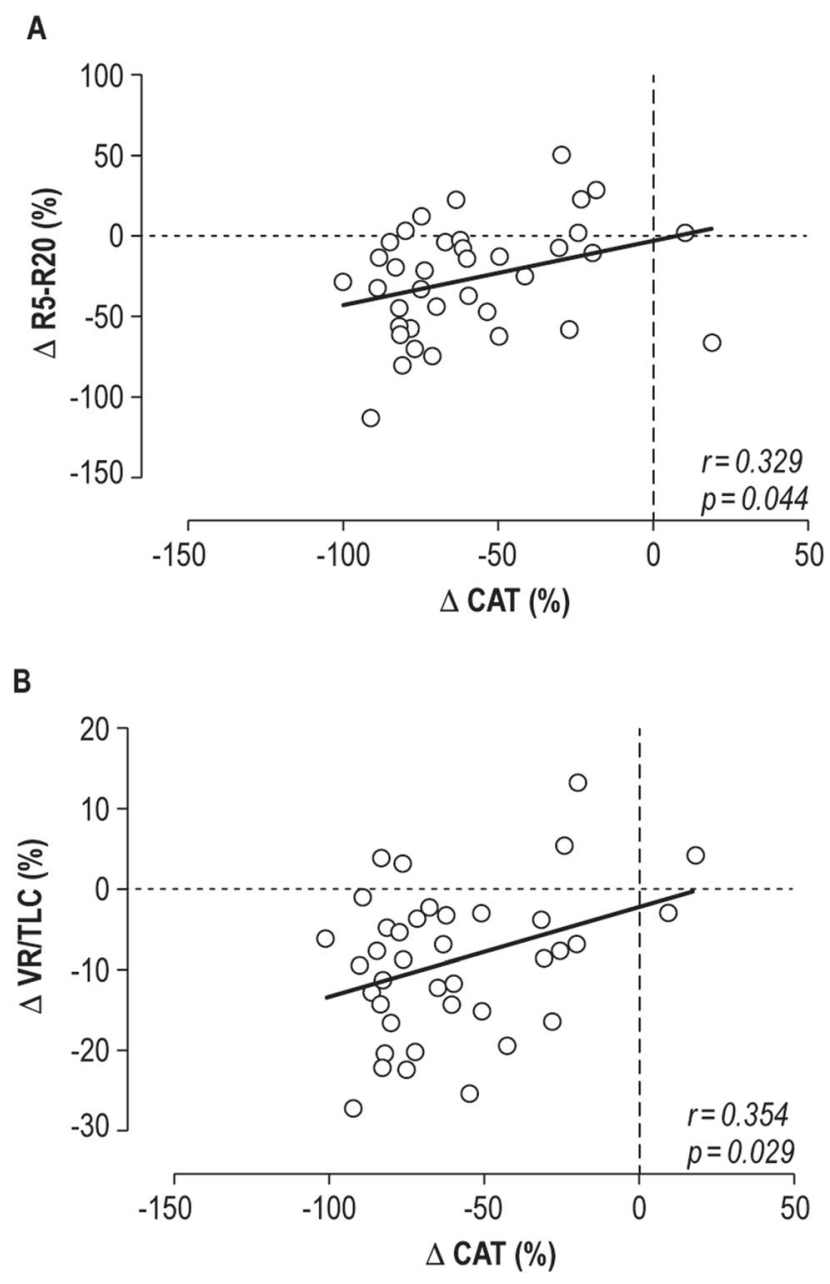

Fig. 2 Relationships between the change after 12 weeks of treatment with BDP/FF in CAT and both R5-R20 values (a) and RV/TLC values $(\mathbf{b})$ in the study population

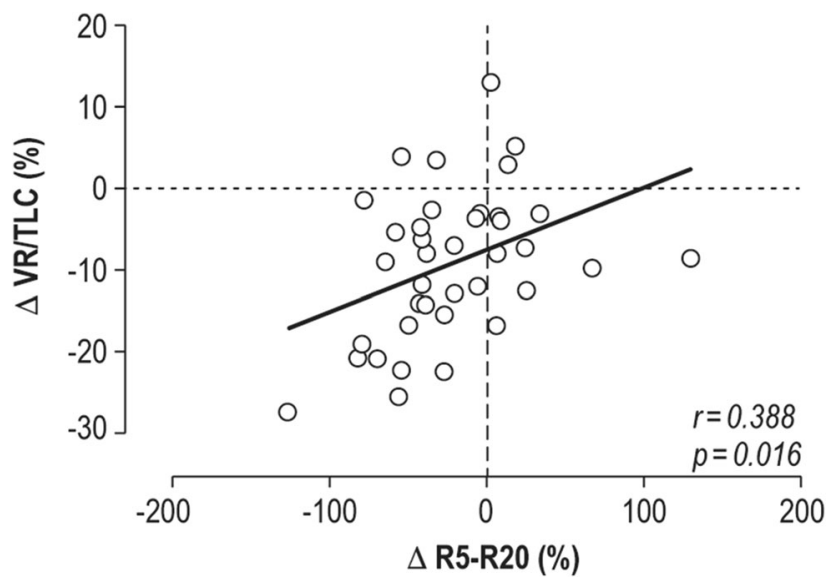

Fig. 3 Relationships between the change after 12 weeks of treatment with BDP/FF in R5-R20 values and RV/TLC values in the study population 
hyperinflation, which represents a functional improvement of the small airway district. In addition, this study provides evidence that the extra-fine formulation of $\mathrm{BDP} / \mathrm{FF}$ improves the impact of the disease in terms of health status, as measured by CAT and mMRC questionnaires. SAD improvement was already detectable after 6 weeks of therapy without any further change at the 12th week, while the symptoms improved throughout the entire study duration. Finally, we confirmed that changes in health status and small airway district function were correlated. We believe that a recent exacerbation cannot explain the high scores seen at baseline since we enrolled patients who had not experienced an exacerbation in the 4 weeks before enrollment.

Our results show significant correlations between the primary outcome and functional findings, which may explain the underlying mechanisms leading to the clinical improvement perceived by patients and likely related to the decrease in pulmonary hyperinflation. The small airways, defined by an internal diameter of less than $2 \mathrm{~mm}$, represent the major site of airflow obstruction in COPD [3, 19, 20]. Several reports showed that SAD (assessed with different functional or radiologic methods) is correlated to the quality of life [5], risk of persistent exacerbations [21], and even poor prognosis [22] in COPD patients. In a previous paper [4], we showed a progressive increase in SAD, moving from GOLD stage $A$ to GOLD stage $D$. Notably, SAD, identified by a rise in R5-R20, got worse with increasing severity of patient's symptoms, resulting in a significant correlation between R5-R20 and CAT scores. Furthermore, Basile and coworkers recently demonstrated a correlation between the extent of the small airways' pathologic involvement, evaluated by IOS, and the relevance of night-time symptoms [23].

Given the importance of reaching and treating the peripheral airways, it is crucial to ensure an adequate peripheral deposition of the inhaled drugs. Inhalation treatment represents the mainstream of COPD treatment; however, the specific intrinsic features of different molecules and how they are combined and formulated must always be considered. The GOLD document [1], as well as other research settings
[24-26], state that extra-fine formulation drugs (i.e., formulations with an $\mathrm{MMAD}<2 \mathrm{mcm}$ ) allow a better lung deposition. Therefore, these formulations are more suitable to reach small airways $[7,27]$.

$\mathrm{BDP} / \mathrm{FF}$ is a drug combination available from the early 2000s, widely used for years with a good safety profile in both COPD and asthma. Among the wide range of inhaled therapies available, its extra-fine formulation ensures a correct drug delivery through the airways, including small airways [26]. BDP/FF has also been formulated in a very innovative DPI device: the NEXThaler ${ }^{\circledR}$. This system has been demonstrated to be intuitive and easy to use, with a simple open-inhale-close technique. Furthermore, it has a triple feedback system, which lets the patient know if the full dose has been correctly inhaled $[26,28]$. Ease of use and device feedback systems are conditions conducive to the therapeutic adherence of the patients. Indeed, our findings showed optimal patient adherence ( $>80 \%$ ) to the BDP/FF NEXThaler ${ }^{\circledR}$ throughout the study.

All patients included in the study had smallairway (SA) functional impairment due to prespecified inclusion criteria. According to our previous experience [4], the prevalence of SAD in COPD is high, except in patients with mild or early disease. In this study, patients had a history of at least one exacerbation and a severe degree of airflow obstruction, which may probably justify a $100 \%$ prevalence of peripheral damage.

The study presents some limitations. First, this is a pilot study, which means that data must be confirmed in a larger population. Secondly, all patients enrolled in the study showed SAD. Future studies in a larger population should enroll patients with the same inclusion criteria but without SAD.

\section{CONCLUSIONS}

This study adds evidence to the topic of pharmacological targeting of the small airways. Our results indicate a link between the effect of the extra-fine BDP/FF DPI combination on this lung region and the improvement in the patient's 
symptoms. A statistically significant improvement of SAD, assessed by changes in R5-R20, was demonstrated after the first 6 weeks of treatment and maintained after 12 weeks. This translated into a significant reduction of hyperinflation. The improvement in CAT scores, which exceeds the minimal clinically significant difference vs. baseline, was statistically correlated to the improvement in SAD parameters, namely R5-R20 and RV/TLC.

Despite not being routinely investigated, small airway measurements can provide supportive information for a tailored therapeutic approach in COPD patients. In this regard, due to its action on both large and small airways, $\mathrm{BDP} / \mathrm{FF}$ delivered in an extra-fine formulation may be considered as a valid combination therapy for a wide population of symptomatic COPD patients who exhibit a high probability of SAD and, consequently, are expected, with effective treatment, to experience a significant functional and clinical improvement.

\section{ACKNOWLEDGEMENTS}

We thank the participants of the study.

Funding. This work and the journal's Rapid Service Fee were supported by an unrestricted grant by Chiesi Farmaceutici, Italy.

Authorship. All named authors meet the International Committee of Medical Journal Editors (ICMJE) criteria for authorship for this article, take responsibility for the integrity of the work as a whole, and have given their approval for this version to be published.

Medical Writing and Editorial Assistance. Editorial assistance was provided by ERA ms. Editorial assistance was funded by Chiesi Farmaceutici, Italy.

Disclosures. Roberta Pisi, Marina Aiello, and Annalisa Frizelli have no conflict of interest to declare. Giuseppina Bertorelli has undertaken research funded by Chiesi Farmaceutici. Alessio Piraino, and Davide Paleari are employees of
Chiesi Farmaceutici. Alfredo Chetta has undertaken research funded by Chiesi Farmaceutici, GlaxoSmithKline, Novartis, and Astra Zeneca.

Compliance with Ethics Guidelines. The study was approved by the Ethics Committee for northern Emilia, Italy (protocol number: 9769; approved on April 5, 2017), and conducted in accordance with good clinical practices and the Declaration of Helsinki. All enrolled patients gave their informed consent.

Data Availability. The datasets used and/or analyzed during the current study are available from the corresponding author on reasonable request.

Open Access. This article is licensed under a Creative Commons Attribution-NonCommercial 4.0 International License, which permits any non-commercial use, sharing, adaptation, distribution and reproduction in any medium or format, as long as you give appropriate credit to the original author(s) and the source, provide a link to the Creative Commons licence, and indicate if changes were made. The images or other third party material in this article are included in the article's Creative Commons licence, unless indicated otherwise in a credit line to the material. If material is not included in the article's Creative Commons licence and your intended use is not permitted by statutory regulation or exceeds the permitted use, you will need to obtain permission directly from the copyright holder. To view a copy of this licence, visit http://creativecommons.org/licenses/by$\mathrm{nc} / 4.0 /$.

\section{REFERENCES}

1. Global Strategy for the Diagnosis, Management and Prevention of COPD, Global Initiative for Chronic Obstructive Lung Disease (GOLD) 2018. Available at http://goldcopd.org

2. Burgel PR. The role of small airways in obstructive airway diseases. Eur Respir Rev. 2011;20(119): 23-33. 
3. Hogg JC, Chu F, Utokaparch S, et al. The nature of small-airway obstruction in chronic obstructive pulmonary disease. N Engl J Med. 2004;350(26): 2645-53.

4. Crisafulli E, Pisi R, Aiello M, et al. Prevalence of small-airway dysfunction among COPD patients with different GOLD stages and its role in the impact of disease. Respiration. 2017;93(1):32-41.

5. Haruna A, Oga T, Muro S, et al. Relationship between peripheral airway function and patientreported outcomes in COPD: a cross-sectional study. BMC Pulm Med. 2010;10:10.

6. Wedzicha JA, Singh D, Vestbo J, et al. Extrafine beclomethasone/formoterol in severe COPD patients with history of exacerbations. Respir Med. 2014;108(8):1153-62.

7. Singh D, Nicolini G, Bindi E, FUTURE (Foster Upgrades TherapeUtic care in REspiratory disease) study group. Extrafine beclomethasone/formoterol compared to fluticasone/salmeterol combination therapy in COPD. BMC Pulm Med. 2014;14:43.

8. Celli BR, MacNee W, Augusti A, ATS/ERS Task Force. Standards for the diagnosis and treatment of patients with COPD: a summary of the ATS/ERS position paper. Eur Respir J. 2004;23(6):932-46.

9. Pellegrino R, Viegi G, Brusasco V, et al. Interpretative strategies for lung function tests. Eur Respir J. 2005;26(5):948-68.

10. Charlson M, Szatrowski TP, Peterson J, Gold J. Validation of a combined comorbidity index. J Clin Epidemiol. 1994;47(11):1245-51.

11. CAT Development Steering Group: COPD Assessment Test-Healthcare Professional User Guide. Issue 4: November 2018. Available from: http:// www.catestonline.org

12. Brooks SM. Surveillance for respiratory hazards. ATS News. 1982;8:12-6.

13. George J, Kong DC, Thoman R, Stewart K. Factors associated with medication nonadherence in patients with COPD. Chest. 2005;128(5):3198-204.

14. Quanjer PH, Tammeling GJ, Cotes JE, et al. Lung volumes and forced ventilatory flows. Report Working Party Standardization of Lung Function Tests, European Community for Steel and Coal. Official Statement of the European Respiratory Society. Eur Respir J Suppl. 1993;16:5-40.

15. Oostveen E, MacLeod D, Lorino H, et al. The forced oscillation technique in clinical practice: methodology, recommendations and future developments. Eur Respir J. 2003;22(6):1026-41.
16. Lipworth BJ, Jabbal S. What can we learn about COPD from impulse oscillometry? Respir Med. 2018;139:106-9.

17. Oppenheimer BW, Goldring RM, Herberg ME, et al. Distal airway function in symptomatic subjects with normal spirometry following World Trade Center dust exposure. Chest. 2007;132(4):1275-82.

18. Kon SS, Canavan JL, Jones SE, et al. Minimum clinically important difference for the COPD Assessment Test: a prospective analysis. Lancet Respir Med. 2014;2(3):195-203.

19. Hogg JC, Macklem PT, Thurlbeck WM. Site and nature of airway obstruction in chronic obstructive lung disease. N Engl J Med. 1968;278(25):1355-60.

20. Yanai M, Sekizawa K, Ohrui T, Sasaki H, Takishima T. Site of airway obstruction in pulmonary disease: direct measurement of intrabronchial pressure. J Appl Physiol (1985). 1992;72(3):1016-23.

21. Han MK, Quibrera PM, Carretta EE, et al. Frequency of exacerbations in patients with chronic obstructive pulmonary disease: an analysis of the SPIROMICS cohort. Lancet Respir Med. 2017;5(8):619-26.

22. Hogg JC, Chu FS, Tan WC, et al. Survival after lung volume reduction in chronic obstructive pulmonary disease: insights from small airway pathology. Am J Respir Crit Care Med. 2007;176(5): 454-9.

23. Basile M, Baiamonte P, Mazzuca E, et al. Sleep disturbances in COPD are associated with heterogeneity of airway obstruction. COPD. 2018;15(4): $350-4$.

24. De Backer W, Devolder A, Poli G, et al. Lung deposition of BDP/formoterol HFA pMDI in healthy volunteers, asthmatic, and COPD patients. J Aerosol Med Pulm Drug Deliv. 2010;23(3):137-48.

25. de Boer AH, Gjaltema D, Hagedoorn P, Frijlink HW. Can 'extrafine' dry powder aerosols improve lung deposition? Eur J Pharm Biopharm. 2015;96: 143-51.

26. Virchow JC, Poli G, Herpich C, et al. Lung deposition of the dry powder fixed combination beclometasone dipropionate plus formoterol fumarate using NEXThaler ${ }^{\circledR}$ device in healthy subjects, asthmatic patients, and COPD patients. J Aerosol Med Pulm Drug Deliv. 2018;31(5):269-80.

27. Calverley PM, Kuna P, Monsó E, et al. Beclomethasone/formoterol in the management of COPD: a randomised controlled trial. Respir Med. 2010;104(12):1858-68. 
28. Voshaar T, Spinola M, Linnane $\mathrm{P}$, et al. Comparing usability of NEXThaler(®) with other inhaled corticosteroid/long-acting combination dry powder inhalers in asthma patients. J Aerosol Med Pulm Drug Deliv. 2014;27(5):363-70. 